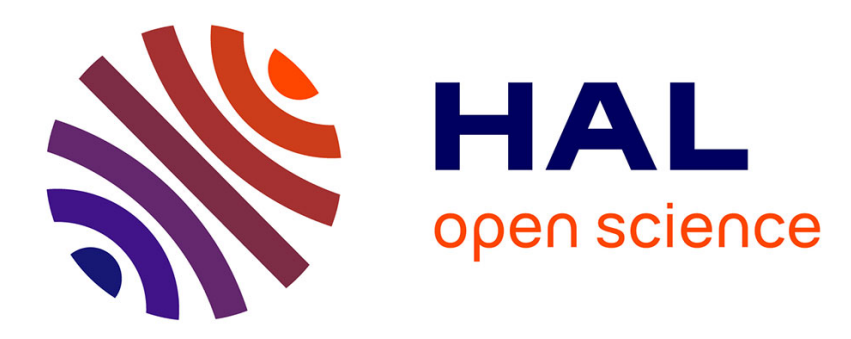

\title{
Transcriptome Profiling of Single Mouse Oocytes
}

\author{
Maud Borensztein, Laurène Syx, Nicolas Servant, Edith Heard
}

\section{To cite this version:}

Maud Borensztein, Laurène Syx, Nicolas Servant, Edith Heard. Transcriptome Profiling of Single Mouse Oocytes. Mouse Oocyte Development, 1818, Springer New York, pp.51-65, 2018, Methods in Molecular Biology, 10.1007/978-1-4939-8603-3_7 . hal-03437409

\section{HAL Id: hal-03437409 \\ https://hal.science/hal-03437409}

Submitted on 19 Nov 2021

HAL is a multi-disciplinary open access archive for the deposit and dissemination of scientific research documents, whether they are published or not. The documents may come from teaching and research institutions in France or abroad, or from public or private research centers.
L'archive ouverte pluridisciplinaire HAL, est destinée au dépôt et à la diffusion de documents scientifiques de niveau recherche, publiés ou non, émanant des établissements d'enseignement et de recherche français ou étrangers, des laboratoires publics ou privés. 
Title

Transcriptome profiling of single mouse oocytes

\section{Running head}

Single oocyte transcriptome

\section{Authors}

Maud Borensztein ${ }^{1,3}$, Laurène Syx ${ }^{1}$, Nicolas Servant ${ }^{2,3}$ and Edith Heard ${ }^{1}$

1, Unité de Génétique et Biologie du Développement, Institut Curie, PSL Research University, CNRS UMR3215, INSERM U934, UPMC Paris-Sorbonne, 26 rue d'Ulm, 75005 Paris, France

2, Institut Curie, PSL Research University, Mines Paris Tech, INSERM U900, 75005, Paris, France

${ }^{3}$, corresponding authors

E-mail: maud.borensztein@curie.fr

nicolas.servant@curie.fr 


\begin{abstract}
Single-cell RNA-sequencing (scRNAseq) enables the detection and quantification of mature RNAs in an individual cell. Assessing single cell transcriptomes can circumvent the limited amount of starting material obtained in oocytes or embryos, in particular when working with mutant mice. Here we outline our scRNAseq protocol, derived from the Tang et al, 2009 protocol, to study mouse oocyte transcriptomes. The method describes the different steps from single cell isolation and cDNA amplification to high-throughput sequencing. The bioinformatics pipeline used to analyse and compare genome-wide gene expression between individual oocytes is then described.
\end{abstract}

\title{
Key words
}

Single-cell RNA-sequencing

Maternal pool

Gene expression

Single-cell bioinformatics pipeline 


\section{Introduction}

Mammalian oocytes and spermatozoa are highly specialized cells, which transmit genetic and epigenetic information to the subsequent generation. Following fertilization, these gametic cells combine to form a totipotent zygote, which will develop into a new organism. During oogenesis, the growing oocytes accumulate mature RNAs (mRNAs), proteins and organelles that will enable early embryonic development. Investigation of the maternal mRNA stock, provided by the mature and quiescent oocyte, is key for a better understanding of early mammalian development, which in turn has major implications for reproductive medicine. Conventional RNA-sequencing (RNAseq) is a widely used approach to study the abundance of mRNAs quantitatively, at a genome-wide level. RNAseq is widely used in the context of hundreds to millions of cells. However, in some situations such as oocytes or early embryos, the scarce amounts of material available render their transcriptomic analyses challenging, in particular in a mutant context. The first single-cell RNA-sequencing (scRNAseq) method was published in 2009 by the Surani laboratory (1-3). This powerful technique enables the study of the transcriptomes of single cells using very limited amounts of material and can reveal cell-to-cell heterogeneity in gene expression.

Here, we describe a complete protocol for the preparation and analysis of transcriptomes of individual mature oocytes collected from pseudo-pregnant mice. The scRNAseq protocol requires collection and isolation in single tube of a single cell and lysis of the cell. At this point, the total mRNA of each oocyte is released into the tube. Retro-transcription of the polyadenylated mRNA into cDNAs and amplification of the cDNAs are directly carried out in the same tube, avoiding unnecessary manipulation/transfers. cDNAs then undergo purification and shearing to generate libraries for high-throughput sequencing. We then present a 
bioinformatics pipeline applied to study gene expression and adapted to the study of single cell transcriptomes.

Importantly, this protocol specifically allows the study of polyadenylated mRNAs, as it is based on the use of polyd(T) primers for retro-transcription (RT) from the 3' UTR of mRNAs. Thus the RT is not full-length but covers on average about $3 \mathrm{~kb}$ from the 3 'UTR. This allows analysis of gene expression but not of differently spliced isoforms. As the small amount of starting material can induce technical variation, amplification quality controls are indispensable to ensure that samples with same amplification yield are analysed. Use of spikein RNAs may be used in parallel for quantification.

The following method has been adapted from the Tang et al, 2009 protocol (1) and subsequent studies (4-6) . Our previous work focused on allele-specific gene expression during mouse early development $(\mathbf{5 , 6})$ and on the maternal to zygotic transition (4). Here, we focus on oocyte transcriptome analysis but similar approaches can be applied to the study of mouse pre-implantation stages from zygote to 4.5 days post-coïtum (late blastocyst) embryos, provided some adjustments, such as the time of embryo collection, or the number of PCR cycles used, depending on whether single blastomeres or single embryos are used. 


\section{Materials}

Bench, pipettes, PCR workstation and thermocycler should be cleaned before the experiment to remove any traces of DNA, DNase and RNase with water followed by nuclease removal reagents. Gloves and lab coat should be worn throughout all bench steps.

\subsection{Oocyte collection}

1. Small plastic tissue culture dishes, sterile

2. Tweezers and scissors for dissection

3. Mouth-controlled holder (see Figure 1a)

4. Thin glass capillaries (see Figure 1b)

5. Micropipette puller (see Figure 1c)

6. Microforge (see Figure 1d)

7. M2 culture medium

8. Hyaluronidase $(0.3 \mathrm{mg} / \mathrm{ml}$ in $\mathrm{M} 2)$

9. Acidic Tyrode's solution, suitable for mouse culture

10. PBS-BSA

Dissolve acetylated BSA (20 mg/ml) in sterile 1X PBS (See Note 1)

11. 0.2 $\mathrm{ml}$ thin-wall PCR tubes, free of RNase, DNase, DNA and PCR inhibitors

12. Binocular microscope

\section{2 cDNA amplification}

1. GeneAmp® 10X PCR Buffer II \& $\mathrm{MgCl}_{2}$ from ThermoFisher Scientific.

2. $10 \% \mathrm{NP} 40$ 
3. SUPERase-in ${ }^{\circledR}(20 \mathrm{U} / \mu \mathrm{l})$ from ThermoFisher Scientific

4. RNase inhibitor ${ }^{\circledR}(40 \mathrm{U} / \mu 1)$ from ThermoFisher Scientific

5. SuperScript® III Reverse Transcriptase from ThermoFisher Scientific

6. 0.1 M DTT included in the SuperScript ${ }^{\circledR}$ III kit

7. $\mathrm{T} 4$ gene 32 protein $(10 \mathrm{mg} / \mathrm{ml})$

8. ExoSAP-IT® from ThermoFisher Scientific

9. $100 \mathrm{mM} \mathrm{dATP}$

10. Terminal Deoxynucleotidyl Transferase, recombinant (15 U/ $\mu 1)$ from ThermoFisher Scientific

11. Rnase $\mathrm{H}(2 \mathrm{U} / \mu \mathrm{l})$

12. 10X Ex Taq polymerase Hot Start ${ }^{\circledR}$ from Takara (Buffer, Taq and $2.5 \mathrm{mM}$ (each) dNTP $\operatorname{mix})$

13. Universal primers, $100 \mu \mathrm{M}$ : AUP1 (5'-NH2-ATA TGG ATC CGG CGC GCC GTC GAC TTT TTT TTT TTT TTT TTT TTT TTT-3') and AUP2 (5'-NH2-ATA TGG ATC CGG CGC GCC GTC GAC TTT TTT TTT TTT TTT TTT TTT TTT-3’, (See Note 2).

14. Thermocycler

15. 0.2 ml thin-wall PCR tubes, free of RNase, DNase, DNA and PCR inhibitors

16. PCR tube centrifuge

\section{3 qPCR check for amplification yield}

1. SYBR-based quantitative PCR kit

2. Real time PCR instrument 
3. Housekeeping gene primers: Hprt (F: ctgtggccatctgcctagt, R: gggacgcagcaactgacatt); Gapdh (F: ccccaacactgagcatctcc, R: attatgggggtctgggatgg); Ppia (F: ttacccatcaaaccattccttctg; R: aacccaaagaacttcagtgagagc) (4)

\section{4 cDNA Purification}

1. PCR purification kit

2. Elution buffer

3. Agarose gel electrophoresis equipment

4. Gel extraction kit

5. Scalpel, sterile.

\subsection{Libraries and sequencing}

1. Qubit ${ }^{\circledR}$ equipment and buffers (or similar quantification equipment)

2. Tapestation ${ }^{\circledR}$ or Bioanalyzer ${ }^{\circledR}$ equipment and buffers (or similar equipment for measurement of DNA size distribution)

3. TE-EDTA low solution

4. Library kit reagents and adapters. This will be chosen in ad equation with the sequencing equipment that will be used.

\subsection{Quality control of the sequencing}

1. Sequencing data (fastq files)

2. Python 2.7 and Biopython tools (SeqIO) 
http://biopython.org/wiki/SeqIO

\subsection{Mapping of sequencing reads}

1. Tophat2 (including the Bowtie2 mapper) (7)

https://ccb.jhu.edu/software/tophat/manual.shtml

2. Mouse reference genome - GRCm38/ mm10 available from the UCSC genome browser (Fasta file)

3. RefSeq annotations available from the UCSC genome browser (GTF file) https://genome.ucsc.edu/cgi-bin/hgTables

\subsection{Counting reads}

1. FeatureCounts (from the Subread package) http://bioinf.wehi.edu.au/featureCounts/

2. RefSeq annotations from the UCSC genome browser (GTF file) https://genome.ucsc.edu/cgi-bin/hgTables

\subsection{Exploratory analysis of sequencing data}

1. R software

https://cran.r-project.org/

2. DESeq2 R package

https://bioconductor.org/packages/release/bioc/vignettes/DESeq2/inst/doc/DESeq2.html

3. Gplots R package 
https://cran.r-project.org/web/packages/gplots/gplots.pdf

\subsection{Differential analysis}

1. R software

2. EdgeR R package

http://bioconductor.org/packages/release/bioc/html/edgeR.html

3. Limma R package

https://www.bioconductor.org/packages/devel/bioc/vignettes/limma/inst/doc/usersguide.pdf

4. Gene Ontology

http://www.geneontology.org 


\section{Methods}

All procedures for oocyte collection and manipulation should be carried out with sterile filter tips and RNase-, DNase-free water, using fresh aliquots for each new experiment.

It is highly recommended to use a PCR workstation (PCR hood) from steps 3.2.1 to 3.2.15 included.

\subsection{Oocyte collection}

1. Collect mature oocytes from female mice (using super-ovulation or matings with vasectomised males) on the appropriate day in M2 culture medium at room temperature. Followed procedures as described in (8).

All animal work should be undertaken under the national ethical guidelines.

2. Remove cumulus cells by culturing oocytes for a few minutes in hyaluronidase at room temperature. Help dissociation by pipetting the oocytes up and down under the binocular microscope, and then rinse oocytes in 3 drops of fresh M2 medium.

3. Transfer the oocytes in acidic Tyrode's solution at room temperature and rinse them in 2 drops of fresh M2 as soon as the zona pellucida removal is observed (See Note 3).

4. Wash the oocytes extensively in 3 drops of PBS-BSA solution.

5. Prepare thin glass capillaries with a pipette-puller and cut them at about $80-100 \mu \mathrm{m}$ in diameter with a Microforge following Manufacter's recommendations for both instruments (see Figure 1 and Note 4).

6. Transfer one oocyte at a time into a clean $0.2 \mathrm{ml} \mathrm{PCR} \mathrm{tube} \mathrm{with} \mathrm{the} \mathrm{smallest} \mathrm{amount} \mathrm{of}$ PBS-BSA. It is important to do a blank tube for each experiment (See Note 5).

7. Quickly put the tube in ice (See Note 6). 


\section{2 cDNA amplification}

From this point on, all steps should be carried out on ice or at $4^{\circ} \mathrm{C}$, unless otherwise stated. Conditions should be RNAse-free until end of step 3.2.7. It is recommended that gloves are changed between each step.

The various steps of single cell picking, cDNA amplification, purification and sequencing are explained in Figure 2. This method is adapted from the Tang et al, 2009 protocol (1).

1. Thaw all the reagents on ice.

2. Dilute the AUP1 primers to $0.5 \mathrm{mM}$ in cold, nuclease-free water $\left(1 \mu \mathrm{l}\right.$ into $199 \mu 1$ of $\left.\mathrm{H}_{2} 0\right)$.

3. Prepare the single cell lysis buffer as below:

\begin{tabular}{|l|l|l|}
\hline Reagents & Final concentration & Volume for 1 reaction \\
\hline PCR Buffer II & $0.9 \mathrm{X}$ & $0.45 \mu \mathrm{l}$ \\
\hline $\mathrm{MgCl}_{2}$ & $1.35 \mathrm{mM}$ & $0.27 \mu \mathrm{l}$ \\
\hline $\mathrm{NP40}$ & $0.45 \%$ & $0.225 \mu \mathrm{l}$ \\
\hline DTT & $4.5 \mathrm{mM}$ & $0.225 \mu \mathrm{l}$ \\
\hline SUPERase-IN@ & $0.18 \mathrm{U} / \mu \mathrm{l}$ & $0.045 \mu \mathrm{l}$ \\
\hline RNase Inhibitor & $0.36 \mathrm{U} / \mu \mathrm{L}$ & $0.045 \mu \mathrm{l}$ \\
\hline $0.5 \mathrm{mM}$ AUP1 primer & $12.5 \mathrm{nmol} / \mathrm{L}$ & $0.125 \mu \mathrm{l}$ \\
\hline dNTPs & $0.045 \mathrm{mM}$ & $0.09 \mu \mathrm{l}$ \\
\hline $\mathrm{H}_{2} 0$ & - & $2.975 \mu \mathrm{l}$ \\
\hline
\end{tabular}

4. Add $4.4 \mu \mathrm{l}$ to each sample tube (on ice) and quickly spin down (See Note 7). 
5. To release mRNA from the oocytes, incubate the tubes in a preheated thermocycler for 90 seconds at $70^{\circ} \mathrm{C}$ and then hold at $4{ }^{\circ} \mathrm{C}$. Quickly spin down the tubes and immediately transfer them to ice. It is important to move to perform step 7 as rapidly as possible.

6. Prepare the Reverse transcription reaction mix during step 5:

\begin{tabular}{|l|l|l|}
\hline Reagents & Final concentration & Volume for 1 reaction \\
\hline SuperScript $₫$ III & $0.9 \mathrm{X}$ & $0.45 \mu \mathrm{l}$ \\
\hline RNase Inhibitor & $1.35 \mathrm{mM}$ & $0.27 \mu \mathrm{l}$ \\
\hline T4 gene 32 protein & $0.45 \%$ & $0.225 \mu \mathrm{l}$ \\
\hline
\end{tabular}

7. Samples then undergo reverse transcription by adding $0.45 \mu 1$ of reaction mix to each tube (final volume is now $5 \mu 1$, see Note 7), followed by an incubation in a preheated thermocycler for 30 minutes at $50^{\circ} \mathrm{C}, 15$ minutes at $70^{\circ} \mathrm{C}$ (enzyme inactivation) and a hold at $4^{\circ} \mathrm{C}$. Quickly spin down the tubes and immediately transfer them to ice.

8. To remove free primers after retro-transcription, add $1 \mu \mathrm{l}$ of ExoSAP-IT® per tube (final volume is now $6 \mu \mathrm{l}$ ), (see Note 7) and incubate in a preheated thermocycler for 30 minutes at $37^{\circ} \mathrm{C}$ followed by 25 minutes at $80^{\circ} \mathrm{C}$ (ExoSAP-IT inactivation) and a $4^{\circ} \mathrm{C}$ hold. Quickly spin down the tubes and immediately transfer them to ice.

9. Prepare reaction mix for 3' poly-A tailing of cDNA just before use.

\begin{tabular}{|l|l|l|}
\hline Reagents & Final concentration & Volume for 1 reaction \\
\hline PCR Buffer II & $1 \mathrm{X}$ & $0.6 \mu \mathrm{l}$ \\
\hline $\mathrm{MgCl}_{2}$ & $1.5 \mathrm{mM}$ & $0.36 \mu \mathrm{l}$ \\
\hline $100 \mathrm{mM}$ dATP & $3 \mathrm{mM}$ & $0.18 \mu \mathrm{l}$ \\
\hline Terminal transferase & $0.75 \mathrm{U} / \mu \mathrm{L}$ & $0.3 \mu \mathrm{l}$ \\
\hline RNase H & $0.1 \mathrm{U} / \mu \mathrm{L}$ & $0.3 \mu \mathrm{l}$ \\
\hline $\mathrm{H}_{2} \mathrm{O}$ & - & $4.26 \mu \mathrm{l}$ \\
\hline
\end{tabular}


10. Add $6 \mu \mathrm{L}$ of the $3^{\prime}$ poly-A tailing mix to each reaction (final volume of $12 \mu \mathrm{L}$ ) and incubate in a preheated Thermocycler for 15 minutes at $37^{\circ} \mathrm{C}$ followed by 10 minutes at $70^{\circ} \mathrm{C}$ for enzyme inactivation and a $4^{\circ} \mathrm{C}$ hold (see Note 7). Quickly spin down the tubes and immediately transfer them to ice.

11. Prepare reaction mix for second strand cDNA synthesis just before use.

\begin{tabular}{|l|l|l|}
\hline Reagents & Final concentration & Volume for 1 reaction \\
\hline Ex Taq Buffer (Takara) & $1 \mathrm{X}$ & $7.6 \mu \mathrm{l}$ \\
\hline dNTPs primers & $1 \mu \mathrm{mol} / \mathrm{L}$ & $7.6 \mu \mathrm{l}$ \\
\hline AUP2 & & $0.76 \mu \mathrm{l}$ \\
$(100 \mu \mathrm{M})$ & & \\
\hline Ex Taq Polymerase & $0.05 \mathrm{U} / \mu \mathrm{L}$ & $0.76 \mu \mathrm{l}$ \\
\hline $\mathrm{H}_{2} 0$ & - & $59.28 \mu \mathrm{l}$ \\
\hline
\end{tabular}

12. Add $76 \mu \mathrm{L}$ of the $2^{\text {nd }}$ strand synthesis mix to each reaction (final volume of $88 \mu \mathrm{L}$ ) and quickly mix without pipetting (see Note 7). Incubate in a thermocycler for 3 minutes at $95^{\circ} \mathrm{C}, 2$ minutes at $50^{\circ} \mathrm{C}, 10$ minutes at $72^{\circ} \mathrm{C}$ and a hold at $4{ }^{\circ} \mathrm{C}$. Quickly spin down the tubes and immediately transfer them to ice.

13. Prepare reaction mix for the PCR amplification step just before use.

\begin{tabular}{|l|l|l|}
\hline Reagents & Final concentration & Volume for 1 reaction \\
\hline Ex Taq Buffer (Takara) & $1 \mathrm{X}$ & $7.6 \mu \mathrm{l}$ \\
\hline dNTPs primers & $1 \mu \mathrm{mol} / \mathrm{L}$ & $7.6 \mu \mathrm{l}$ \\
\hline AUP1 & & $0.76 \mu \mathrm{l}$ \\
$(100 \mu \mathrm{M})$ & & \\
\hline Ex Taq Polymerase & $0.05 \mathrm{U} / \mu \mathrm{L}$ & $0.76 \mu \mathrm{l}$ \\
\hline
\end{tabular}




\begin{tabular}{|l|l|l|}
\hline $\mathrm{H}_{2} \mathrm{O}$ & - & $59.28 \mu \mathrm{l}$ \\
\hline
\end{tabular}

14. Add $76 \mu \mathrm{L}$ of PCR amplification mix to each reaction (final volume of $164 \mu \mathrm{L}$ ) and quickly mix without pipetting (see Note 7).

15. Aliquot each reaction into $2 \mathrm{PCR}$ tubes of $82 \mu \mathrm{L}$ each.

16. Incubate in a thermocycler for 3 minutes at $95^{\circ} \mathrm{C}$, followed by $20 \mathrm{PCR}$ cycles $\left(95^{\circ} \mathrm{C}, 30\right.$ seconds; $67^{\circ} \mathrm{C}, 1$ minute; $72^{\circ} \mathrm{C}, 6$ minutes plus 12 additional seconds per cycle), and a hold at $4^{\circ} \mathrm{C}$ (see Note 8$)$.

17. Pool the 2 PCR tubes into one for each sample.

18. PCR products can now be stored at $-80^{\circ} \mathrm{C}$ for 6 months.

\section{3 qPCR check for amplification yield}

Quality control of the amplified cDNA is a critical step before pursuing the experiment to ensure that only good quality samples are further processed. The amplification yield is validated based on the expression of three different housekeeping genes (Hprt, Gapdh and Ppia), using a SYBR-based quantitative PCR. It is critical to analyse samples that give the same yield of cDNAs following amplification when compared to other samples from the same developmental stage/background.

This step should be adapted for the Real-Time PCR instrument and kit used.

An aliquot of the PCR products should be diluted at 1:50 in nuclease-free water to be used as a template for the Real-Time PCR runs for quantification of housekeeping genes.

\section{4 cDNA purification}


1. Purify the remaining undiluted PCR product (about $160 \mu \mathrm{L}$ ) with a PCR purification kit. Elute in $30 \mu \mathrm{L}$ of nuclease-free water or elution Buffer (See Note 9). The purified PCR product can be stored at $-80^{\circ} \mathrm{C}$ for 6 months.

2. To remove primers dimers and size-select the amplified cDNA, load all purified PCR products on a $2 \%$ agarose gel alongside an appropriate size standard (See Note 10).

3. Run the gel until the bromophenol blue dye marker is $2-3 \mathrm{~cm}$ from the well.

4. Excise cDNA from the size range of $0.5-5 \mathrm{~kb}$ with a sterile sharp scalpel. Clean the scalpel with milli-Q water between each sample.

5. Purify with a gel extraction kit. Elute the cDNA in 50ul elution buffer (See Note 9).

6. The purified product can be stored at $-80^{\circ} \mathrm{C}$ for 6 months.

\subsection{Libraries and sequencing}

1. Measure the concentration of purified DNA products by Qubit@ or equivalent.

2. Shear $300 \mathrm{ng}$ of double strand cDNA (diluted into TE-EDTA low solution) into fragments with an average peak of $200 \mathrm{bp}$ by sonication. Follow the manufacturer's recommendations in accordance to the library preparation kit that will be used. (See Note 11).

3. Measure the size distribution after shearing with Tapestation ${ }^{\circledR}$ or equivalent equipment.

4. Prepare the library and use adaptors to multiplex the different samples, following the manufacturer recommendations.

5. Send the samples for high-throughput sequencing.

\subsection{Quality control of sequencing}


We then describe the bioinformatics pipeline applied to study gene expression in single oocyte transcriptomes (See Figure 3).

\section{7}

In this Method, bioinformatics analysis is performed on libraries sequenced on Illumina Hiseq 2500 instrument, providing single-end, non-stranded 50bp reads. Quality control is applied on the raw sequencing data following the particularities of the single-cell RNAseq protocol described above.

Overall sequencing quality control can be first performed using standard tools such as the FastQC sotfware (https://www.bioinformatics.babraham.ac.uk/projects/fastqc/).

Reads characterized by at least one of the following criteria must be discarded:

1. More than $50 \%$ of low quality bases (Phred score $<5$ )

2. More than $5 \%$ of $\mathrm{N}$ bases

3. At least $80 \%$ of AT rate

4. More than $30 \%$ of continuous A and/or T.

This read filtering can be performed using the Python programming language and the BioPython module that eases the manipulation of sequencing data into the Python framework.

\subsection{Mapping of sequencing reads}

1. Mouse reference genome $(\mathrm{GRCm} 38 / \mathrm{mm} 10)$ and associated RefSeq annotations file (GTF format) should be downloaded from the UCSC Table Browser (See Note 12). 
2. Align reads to the mm10 genome using the Tophat 2 software (9) with the following parameters: “--max-multihits 1 --transcriptome-max-hits 1 --no-coverage-search”. Thereby, only unique alignments with less than 2 mismatches are reported for downstream analyses. Note that other RNAseq reads mappers can also be used at this step.

\subsection{Counting reads}

From the aligned data (BAM files), generate count tables of gene expression using the RefSeq gene model annotation (GTF format) and the featureCounts tool (10), with default parameters (i.e "-s unstranded -F GTF/GFF -t exon -g gene_id").

See Note 13

\subsection{Exploratory analysis of counts table}

The first step of most bioinformatics analyses is to visually explore the sample relationships based on their gene expression profile without any a priori knowledge. For this:

1. The raw counts matrix is first filtered to remove all genes with no or nearly no counts in all samples (See Note 14).

2. The filtered raw counts matrix is then transformed using the regularized-logarithm transformation (or rlog) function implemented in the DESeq2 R package (11). Briefly, this transformation allows stabilizing the variance across the mean expression value before exploratory analysis.

3. Hierarchical clustering (Pearson correlation distance - Ward method) of samples based on their gene expression is performed using the hclust function implemented in the gplots $\mathrm{R}$ 
package. See Figure 4 for an example of Pearson correlation distance between two scRNAseq produced in the same experiment, from C57BL6/J oocytes.

\subsection{Differential analysis}

One of the main goals of RNAseq studies is to identify differentially expressed genes between two or more conditions, such as mutant and wild-type samples, cell-to-cell heterogeneity, developmental (or other) dynamics.

1. Using raw counts matrix, all genes (in rows) with zero or very low counts in all samples are removed (See Note 14).

2. Trimmed Mean of M-values (TMM) normalization is performed on filtered count matrix using the EdgeR package. This normalisation technique is well adapted for comparative studies.

3. The Voom transformation is then applied to the normalized matrix and the detection of differentially expressed genes is performed with the Limma $\mathrm{R}$ package (See Note 14). Multiple testing adjustment is performed using the Benjamini-Hochberg procedure. Genes with an adjusted p-value lower than 5\% are usually considered as significant.

4. Differentially expressed genes with an absolute value of logFoldChange higher than 1 can then be used to further explore the involved biological pathways. 


\section{Notes}

1. PBS-BSA solution should be aliquoted in $0.5 \mathrm{ml}$ sterile tubes and kept at $-20^{\circ} \mathrm{C}$. New aliquots should be thawed out for each sample collection.

2. Primers should be purified by HPLC and their 5' are modified by an amino C6 dT.

3. Oocytes should be cultured in acidic Tyrode's solution for no more than few minutes and kept in suspension by pipetting. This avoids oocytes sticking to the bottom of the plastic dish.

4. For convenience, thin glass capillaries can be prepared in advance. They should then be kept in a clean box to avoid any contamination. The diameter of capillaries should be adapted if used for single blastomeres (4-cell stage and onwards) or single embryos (mid and late blastocysts).

5. The amount of PBS-BSA during the oocyte transfer is critical and should be kept to a minimum $(0.1-0.5 \mu 1)$. Another critical aspect is to collect only good samples based on their normal morphology (if applicable in the case of mutants).

Furthermore, a blank tube should be made for each experiment by transferring the same amount of PBS-BSA without oocyte into a new tube. PBS-BSA used for the blank should be taken from the same drop in which oocytes were kept.

6. After sample collection, tubes can be kept at $-80^{\circ} \mathrm{C}$ for no more than 2 months. If so, on the day of cDNA amplification, keep the tubes on ice when removed from the $80^{\circ} \mathrm{C}$ freezer and add the lysis solution to the tubes as rapidly as possible (see Methods 3.2.4).

7. Avoid pipetting up and down into the tube to limit loss of RNA or cDNA molecules.

8. The number of PCR cycles should be optimized if developmental stages other than oocytes, zygotes and 2-cell stage embryos are being analysed. Increase or decrease the 
number of PCR cycles for single blastomeres or single embryos respectively from the 4-cell stage onwards.

9. Manufacturer's recommendations should be followed.

10. Carefully clean the electrophoresis equipment with water before use and interspace each sample on the agarose gel to avoid cross-contamination.

11. For sonication with a Covaris E220 ultrasonicator, we diluted 300ng DNA into TEEDTA low to obtain a final volume of $50 \mu 1$ and transferred it into microTUBES AFA Fiber Pre-Slit Snap-Cap from Covaris@. We then used the following settings: peak power 175, duty factor 10, cycles/burst 200, duration 200 seconds, temperature $6^{\circ} \mathrm{C}$.

12. In this example, our oocytes are from the C57BL6/J background. RefSeq files should be chosen as a function of the mouse strain used.

13. Parameters should be adapted according to the sequencing method (e.g. single-end or paired-end, un-stranded or stranded or reverse stranded...).

14. Thresholds should be tested and adapted to the protocol used.

15. At this stage, the statistical model must be carefully defined according to the experimental design. Statistical expertise should always be sought prior to performing the experiment and the statistical model should be designed and/or validated by a statistician. 


\section{References}

1. Tang F, Barbacioru C, Wang Y, et al. mRNA-Seq whole-transcriptome analysis of a single cell. Nat Methods. 2009;6(5). doi:10.1038/NMETH.1315.

2. Tang F, Barbacioru C, Nordman E, et al. RNA-Seq analysis to capture the transcriptome landscape of a single cell. Nat Protoc. 2010;5(3):516-535. doi:10.1038/nprot.2009.236.

3. Tang F, Lao K, Surani MA. Development and applications of single-cell transcriptome analysis. Nat Methods. 2011;8(4). doi:10.1038/NMETH.1557.

4. Ancelin K, Syx L, Borensztein M, et al. Maternal LSD1/KDM1A is an essential regulator of chromatin and transcription landscapes during zygotic genome activation. Elife. 2016;5(e08851). doi:10.7554/eLife.08851.

5. Borensztein M, Syx L, Ancelin K, et al. Xist -dependent imprinted X inactivation and the early developmental consequences of its failure. Nat Struct Mol Biol. 2017;24(3):226-233. doi:10.1038/nsmb.3365.

6. Borensztein M, Okamoto I, Syx L, et al. Contribution of epigenetic landscapes and transcription factors to $\mathrm{X}$-chromosome reactivation in the inner cell mass. Nat Commun. 2017;8:1-14. doi:10.1038/s41467-017-01415-5.

7. Kim D, Pertea G, Trapnell C, Pimentel H, Kelley R, Salzberg SL. TopHat2: accurate alignment of transcriptomes in the presence of insertions, deletions and gene fusions. Genome Biol. 2013;14(4):R36. doi:10.1186/gb-2013-14-4-r36.

8. Nagy A, Gertsenstein M, Vintersten K, Behringer R. Manipulating the Mouse Embryo: a laboratory manual. In: Cold Spring Harbor Laboratory Press. ; 2003.

9. Trapnell C, Pachter L, Salzberg SL. TopHat: Discovering splice junctions with RNASeq. Bioinformatics. 2009;25(9):1105-1111. doi:10.1093/bioinformatics/btp120.

10. Liao Y, Smyth GK, Shi W. Sequence analysis featureCounts : an efficient general 
purpose program for assigning sequence reads to genomic features. Bioinformatics. 2014;30(7):923-930. doi:10.1093/bioinformatics/btt656.

11. Love MI, Huber W, Anders S. Moderated estimation of fold change and dispersion for RNA-seq data with DESeq2. Genome Biol. 2014;15(550):1-21. doi:10.1186/s13059014-0550-8. 


\section{Figure legends}

Figure 1. Equipment setup for the mouth-controlled holder and thin glass capillaries for single oocyte transfer to PCR tube. (a) Mouth-controlled holder made of an aspirator tube, a mouth adaptor and a filter tip. (b) Thin glass capillaries of about 80-100 $\mu \mathrm{m}$ in diameter. (c) Micropipette puller is used to pull glass capillaries. (d) Microforge is used to cut and firepolished the capillaries at the appropriate diameter.

Figure 2. Methods summary of pipeline. After collection of mature oocytes and transfer of each single cell to a PCR tube, total mRNAs are released in the tube and retro-transcribed. cDNA, after poly-A tailing, are double-stranded and then amplified by PCR. Amplified cDNAs are then purified and size selected on agarose gel. Libraries are made with the sheared DNA and then deep-sequenced.

Figure 3. Bioinformatics pipeline for single-cell RNA-sequencing. Raw data obtained after sequencing (RNAseq read files) must be put through a quality check in order to remove poor quality reads. Reads are aligned to the mouse reference genome and their sum is counted per gene. Exploratory and differential expression analysis can then be assessed to study unsupervised relationships and differentially expressed genes between samples. In red is highlighted the software used for each step.

Figure 4. Example of gene expression correlation (Pearson correlation) between two different oocytes from C57BL6/J background. scRNAseq analysis showed more than $98 \%$ of correlation in gene expression, genome-wide, between two independent oocytes, amplified in parallel. 